\title{
Electromagnetic Heating Effects in Power Distribution Cables under Different Operating Conditions
}

\author{
M. Eladawya, ${ }^{\mathrm{*}}$, and I.A. Metwallyb \\ a Electrical Engineering Department, Faculty of Engineering, Mansoura University, Mansoura 35516, Egypt. \\ ${ }^{b}$ Department of Electrical and Computer Engineering, College of Eng. Sultan Qaboos University, Muscat 123, Oman.
}

Received 8 April 2018; Accepted 22 April 2018

\begin{abstract}
This paper presents a finite element simulation by COMSOL Multiphysics package to investigate the temperature distribution inside three-phase, three-core, $33 \mathrm{kV}$ underground power cables (UGC) through a coupled electromagnetic-thermal modelling. The simulations are very controlled and fine realistic details can be added to the model such as the temperature conductivity dependence of any metallic layer and armour permeability. Distributions of magnetic field, current density, resistive losses and temperature inside UGC different layers are calculated at different operating conditions. The exponential increase in conductor temperature with increasing the conductor current limits the single-phasing operation of such cables. Therefore, they must be derated, otherwise their lifetime will be reduced exponentially. Finally, the effect of current harmonics on the temperature distribution inside the insulation material and hence its lifetime is calculated using MATLAB. It is found that higher steady-state conductor temperatures are expected for cables with larger conductor cross-sectional areas, using aluminium core rather than copper, or using 6-pulse rectifiers rather than a higher pulse types.
\end{abstract}

Keywords: Power cables; Heating; Electromagnatic modelling.

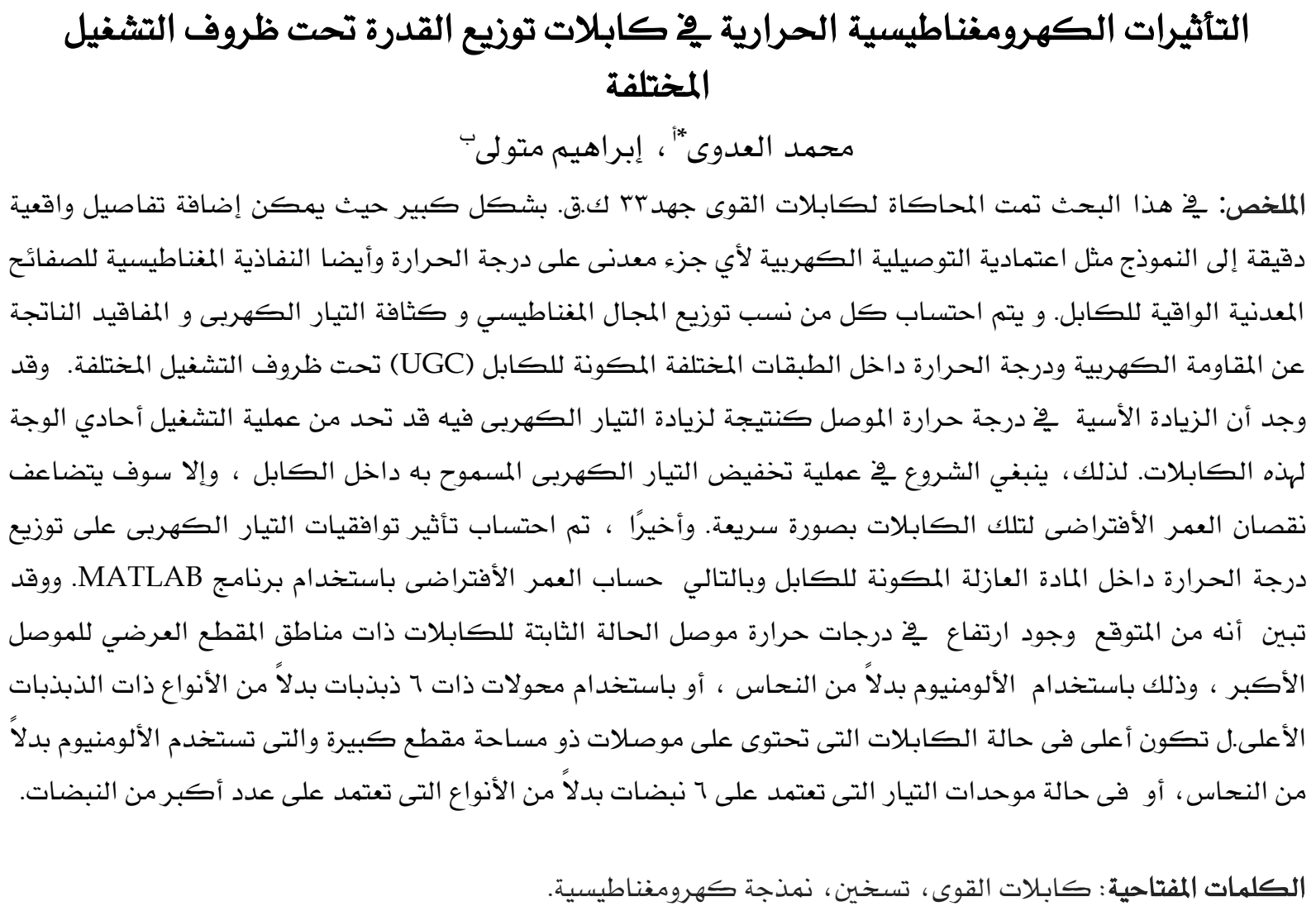




\section{Introduction}

Significant growing demand of electrical power has been a great challenge in transmission and distribution systems over the years due to the progressive increase of power consumers. Underground cables (UGCs) are widely used by utilities to transmit and distribute electric power at corresponding different voltage levels from generating stations to urban areas in order to provide an environment-friendly and reliable pathway for this power flow. Consequently, this leads the utilities to evaluate the performance of UGCs considering their design, reliability, lifetime, manufacturing and installation costs. Sufficiently accurate modelling is, in general, more difficult to obtain for UGCs than that for overhead transmission lines due to the complexity of geometrical configuration of UGCs, which leads to high sensitivity of cable parameters to errors. Advanced diagnostic and simulation techniques can be used for accurate assessment of UGC insulation condition and consequently monitoring the probable reasons related to failure process of the insulation system (Moore 1997).

Numerical modelling (Huang et al. 2015) of UGCs provides, especially when calibrated with empirical results, an attractive tool to study the electromagnetic heating effect inside them, improve design, identify the dielectric response of the insulation system and determine temperature distribution through the different layers of the UGC in order to reduce manufacturing costs and improve design. However, the dielectric material of medium voltage cables is usually polymeric materials. One of the major problems, which shortens its lifetime, is the increase of its operating temperature than the maximum permissible one. However, this increase of temperature may arise from any imposed harmonics on the maximum allowable current carrying capacity (ampacity) or any surrounding heat source such as other neighbouring UGCs (Anders et al. 1999). The simulation of the coupled electromagnetic-thermal model of UGCs can provide a simple tool to identify any increase in temperature due to the normal and abnormal operating conditions, which could be harmful and may shorten its lifetime over the long-term operation.

Accordingly, the purpose of this work is to provide a numerical model of electromagnetic heating effect in a three-phase medium-voltage UGC under different operating conditions through finite element simulation using COMSOL Multiphysics package (2013). This model allows realistic details to be added and to study their effects on the temperature distribution inside different UGC layers Moreover, different modes of operations can be studied such as unintended single-phasing operation of a three-phase UGC. Firstly, these calculations are considered for pure sinusoidal current waveforms. Thereafter, the investigations are followed by considering the effect of harmonic contents on the temperature rise and consequently the lifetime of UGCs.

\section{Transient Electromagnetic Heating Modelling}

For a relatively uniform surrounding environment and when the cable length is much larger than its overall diameter, a twodimensional model can be used for this simulation as shown in Fig. 1. To study the electromagnetic heating characteristics in UGCs, COMSOL Multiphysics package (2013) is used to build a model for a $33 \mathrm{kV}, 240 \mathrm{~mm}^{2}$, threephase, screened three-core belted armoured UGC buried at $0.8 \mathrm{~m}$, which represents the mainstream of installed UGCs (SAOG). In general, heat transfer mechanisms comprise conduction, convection and radiation. When the cables are directly buried, heat transfer mainly depends on the conduction mechanism due to soil thermal characteristics and burial depth, whereas convection due to air and solar radiation effect can be ignored for instance (Moore 1997).

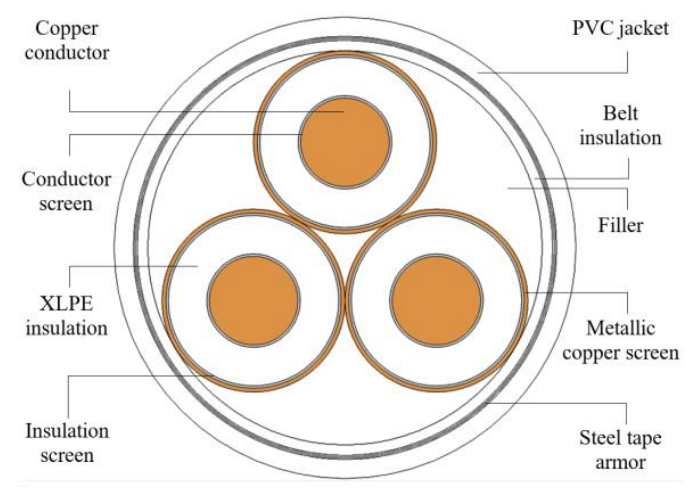

Figure 1. A typical 2D cross section of threephase screened three-core belted underground cable. 
Consequently, the time-dependent heat transfer equation has the following form:

$$
Q(T, A)=\rho C_{p} \frac{\partial T}{\partial t}-\nabla \cdot(K \nabla T)
$$

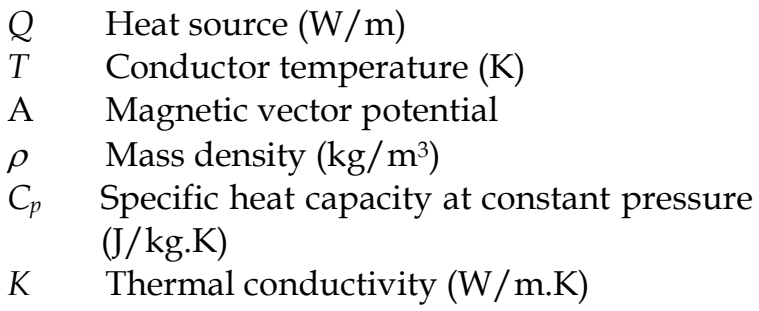

The heat source $Q$ generally incorporates any resistive heating and magnetic losses through the Poynting's theorem (Kovetz 1990). This can be calculated from the definition of magnetic vector potential and the application of MaxwellAmpere's law taking into account the temperature dependence of electrical conductivity as follows:

$$
\begin{aligned}
& \sigma=\left(\rho_{0}\left(1+\alpha\left(T-T_{0}\right)\right)\right)^{-1} \\
& J=j \omega \sigma A+\omega^{2} \varepsilon A+J_{e} \\
& J_{e}=\left(j \omega \sigma-\omega^{2} \varepsilon\right) A+\nabla \times\left(\mu^{-l} \nabla \times A\right) \\
& Q=\frac{1}{2} \operatorname{Re}\left(J \cdot(-j \omega A)^{*}\right)+\frac{1}{2} \operatorname{Re}\left(j \omega B \cdot H^{*}\right)
\end{aligned}
$$

where:

$$
\begin{array}{ll}
\sigma & \text { Electrical conductivity }(\mathrm{S} / \mathrm{m}) \\
\rho_{0} & \text { Reference resistivity }(\Omega . \mathrm{m}) \\
a & \text { Temperature coefficient of resistivity }\left(\mathrm{K}^{-1}\right) \\
T_{0} & \text { Reference temperature }(293 \mathrm{~K}) \\
J & \text { Total current density }\left(\mathrm{A} / \mathrm{m}^{2}\right) \\
J_{e} & \text { Current density due to an external source } \\
\left(\mathrm{A} / \mathrm{m}^{2}\right) \\
\omega & \text { Angular frequency }(\mathrm{rad} / \mathrm{s}) \\
\varepsilon & \text { Permittivity }(\mathrm{F} / \mathrm{m}) \\
\mu & \text { Permeability }(\mathrm{H} / \mathrm{m}) \\
\operatorname{Re} & \text { Real operator of complex quantity }
\end{array}
$$

Moreover, for a fixed frequency and relative phase shift of $120^{\circ}$ between phases, the resulting three-phase currents distribution depends on the conductivity and permeability of each material layer of UGC through both skin and proximity effects.

Boundary conditions are critically important with the equations describing the electromagnetic heating model. Since the magnetic field can extend outside the UGC with copper armour tape, a relatively large domain of soil outside the cable has been modelled. However, the size of soil region was chosen by studying progressively larger region in order to reduce the error difference to be smaller than 104 between the two consecutive simulations when increasing the region size. The magnetic potential at the soil boundaries, which sets the tangential components of the magnetic potential is considered to be zero as follows (COMSOL 2013):

$n \times A=0$

where $n$ is the normal component vector. In this model, the ambient soil temperature at the ground level is set as $20^{\circ} \mathrm{C}$ and the calorific flow continuity has been considered at the separation surface between any two different materials as follows (COMSOL 2013):

$K_{1} \frac{\partial T}{\partial n}=K_{2} \frac{\partial T}{\partial n}$

where $K_{1}$ and $K_{2}$ are the thermal conductivities of the two adjacent media 1 and 2, respectively. In this model, the ambient soil temperature at the ground level is set as $20^{\circ} \mathrm{C}$ and the calorific flow continuity has been considered at the separation surface between any two different materials.

In order to increase the computation accuracy, a non-uniform mesh refinement process is a base for further analysis whereas the mesh of minimum size near the boundaries and hence higher gradients significantly influence the calculation accuracy. Furthermore, the mesh of minimum size is subjected to a parametric study in order to reduce the error difference between two consecutive runs of simulation. However, this difference was smaller than $10^{-4} \%$ without any considerable impact on the calculated field or thermal distributions in the bulk of materials (Huang et al. 2015).

\section{Modelled Three-Phase Three-Core XLPE Insulated UGC}

Table 1 summarizes the geometrical characteristics of a $33 \mathrm{kV}$, three-phase, threecopper conductor UGC shown in Fig. 1. However, the insulation systems mainly comprise three parts; conductor insulation, belt 
insulation and the filling material in the interstice between the cores. The conductor insulation is based on cross-linked polyethylene insulation (XLPE) while the filler and belt insulation is based on ethylene propylene rubber (EPR). For the purpose of calculation, it is assumed that the conductor surrounding insulation materials are uniform and the conductor has a smoothed and circular cross section. This is a valid postulation since in the case of stranded conductor; a semiconductor shield is applied to regulate any concentration of stress that may result due to stranded wires.

In UGC, semiconducting materials are widely used to mitigate the electrical stress at the interfaces between the conductor and insulation and between the insulation and metallic copper screen layer as shown in Fig. 1. Typically, ethylene vinyl acetate (EVA) and ethylene ethyl acrylate copolymers (EEA) are widely adopted as semiconducting screens (Moore 1997). The metallic sheath in such cable (SAOG) consists of a double steel tape armour covered by an outer PVC jacket to protect against environmental hazard. Moreover, the relative permeability of steel tape armour is of interest due to its effect on the magnetic field distribution and consequently its thermal performance. Some of the physical parameters of the materials used for this calculation are summarized in Table 2.

Soil characteristics, such as its type and moisture content, significantly control heat dissipation outside UGC surface. When UGC surface temperature exceeds its critical value, a boundary zone of soil surrounding the cable starts to dry out resulting in higher thermal resistivity than prior to drying out (Anders 2005; Gouda et al, 2010). This in turn increases the temperature of the cable sheath. However,

Table 1. Geometrical characteristics of a $33 \mathrm{kV}, 240 \mathrm{~mm}^{2}$, three-phase, three-core copper conductor, XLPE insulated, steel armored cable (SAOG).

\begin{tabular}{lll}
\hline \multicolumn{1}{c}{ Quantity } & Value & Unit \\
\hline Conductor diameter & 18.4 & $\mathrm{~mm}$ \\
Nominal insulation thickness & 8.0 & $\mathrm{~mm}$ \\
Nominal separation sheath thickness & 2.2 & $\mathrm{~mm}$ \\
Nominal steel tap thickness & 0.8 & $\mathrm{~mm}$ \\
Nominal outer PVC sheath thickness & 4.0 & $\mathrm{~mm}$ \\
Approximate cable overall diameter & 86.0 & $\mathrm{~mm}$ \\
DC resistance of conductor at $20^{\circ} \mathrm{C}$ & 0.0754 & $\Omega / \mathrm{km}$ \\
$\begin{array}{l}\text { Current rating for } 0.8 \mathrm{~m} \text { laying depth and } \\
\text { thermal resistivity of soil }=1.5^{\circ} \mathrm{C} \mathrm{m} / \mathrm{W} .\end{array}$ & 431 & $\mathrm{~A}$ \\
\hline
\end{tabular}

Table 2. Physical constants of the materials used in UGC (Moore 1997).

\begin{tabular}{lcccc}
\hline Material & $\begin{array}{c}\text { Density } \\
\left(\mathbf{k g} / \mathbf{m}^{\mathbf{3}}\right)\end{array}$ & $\begin{array}{c}\text { Specific heat } \\
(\mathbf{J} / \mathbf{( k g . K )})\end{array}$ & $\begin{array}{c}\text { Relative } \\
\text { permittivity }\end{array}$ & $\begin{array}{c}\text { Thermal } \\
\text { conductivity } \\
(\mathbf{W} /(\mathbf{m} . \mathbf{K}))\end{array}$ \\
\hline Copper & 8700 & 385 & 1 & 400 \\
EVA & 925 & 1400 & 2.25 & 0.34 \\
XLPE & 930 & 1900 & 2.3 & 0.286 \\
EPR & 860 & 2000 & 2.35 & 0.286 \\
steel & 7780 & 460 & 1 & 18 \\
PVC & 1760 & 900 & 2.9 & 0.2 \\
Soil & 770 & 1500 & 4 & 0.667 \\
\hline
\end{tabular}


the rest of soil has uniform thermal resistivity outside the dry zone corresponding to its moisture content. For simplicity, the entire soil surrounding the cable is considered to have uniform thermal characteristics prior to drying out. For more details see (Anders 2005; Eladawy 2017).

\section{Results and Discussion}

\subsection{Signatures of Magnetic Field and Resistive Losses}

Maxwell's equations in frequency domain govern the electromagnetic field diffusion inside the cable and the surrounding soil because of current application to the conductors. With the specified magnitude of each carrying current and phase angles of $0^{\circ}$ for upper, $120^{\circ}$ for right and $-120^{\circ}$ for left conductors in the case of power frequency range $(50 \mathrm{~Hz})$, the total current density is based on the source current and induced eddy current densities. As a reference case for simulation, a constant relative permeability of the steel tape armour is considered to be 500 .

Figure 2a shows a surface plot of current density inside the metallic parts of the UGC. A non-uniform current density distribution is observed in the adjoining conductors due to both skin and proximity effects of the flowing core currents that induce eddy currents in all the metallic parts of the cable. However, the non-uniform current density inside the copper conductors (cores) and their metallic copper screens can be attributed to the current displacement phenomenon which diverges the currents inside the adjacent conductors. It can be observed that the maximum eddy current density takes place inside the three copper conductors followed by the closest individual copper sheath boundaries to the steel tape armour with $120^{\circ}$ phase shift of each (Metwally 2010). This constant phase shift has the effect of transferring constant power over each current cycle.

Figure $2 \mathrm{~b}$ shows a surface plot of the resistive losses due to these current distributions with proportional arrow representation of the surrounding magnetic field intensity inside the cable and the surrounding soil. It is clear that the resistive losses follow the same trend of current distribution as observed in Fig. 2a. However, its value depends on the current distribution and the specific resistance of the metallic parts and its variation with tempara-

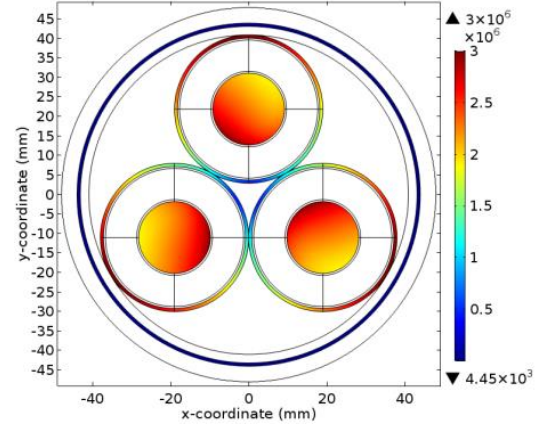

(a) Surface plot of current density $\left(\mathrm{A} / \mathrm{m}^{2}\right)$.

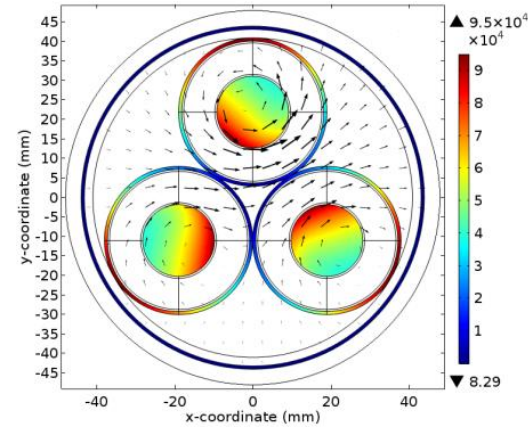

(b) Surface plot of resistive losses $\left(\mathrm{W} / \mathrm{m}^{3}\right)$ with proportional arrow direction of magnetic field intensity $(\mathrm{A} / \mathrm{m})$.

Figure 2. Distribution of current density and corresponding resistive losses of the metallic parts in medium voltage UGC after $3000 \mathrm{~h}$ of simulation.

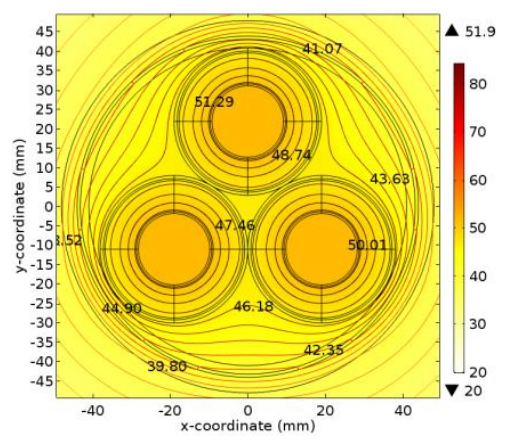

(a) $t=10 \mathrm{~h}$.

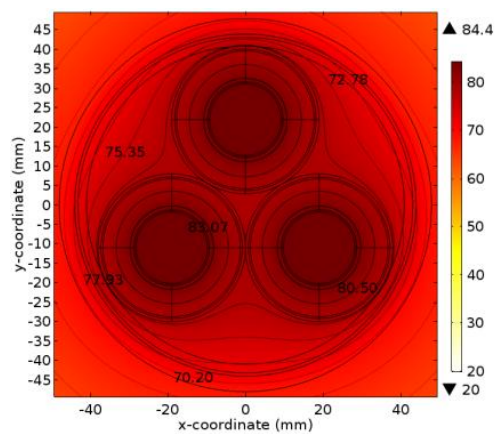

(b) $t=3000 \mathrm{~h}$.

Figure 3. Surface plot and level contours of temperature distribution inside UGC at two times of simulation until steady-state thermal equilibrium. 
ture. It is worth mentioning that the losses in the copper screen (sheath) of each core also depend on the bonding arrangement (Moore 1997). This issue is not currently studied in this paper.

\subsection{Transient Temperature Evolution}

Thermal behaviour of UGC has received a lot of attentions from numerous manufacturers and researchers in order to improve cable design and electric installation guidelines for safe ampacity calculation of conductor and reliable operation of the constructive insulation material. However, this behaviour is a robust function of loading current, burial depth, soil thermal characteristics, skin and proximity effects, sheath and armour losses, internal and external thermal resistances and current distribution inside the cable parts (Metwally 2012; Anders et al. 1999; IEC 60287 1994, Anders 2005). According to IEC standard method for the thermal rating of UGC (IEC 60287 1994), several approximations and simplifications of this function may lead to underestimation of more than $5 \%$ in the calculated maximum continuous conductor temperature (Sedaghat $e t$ al. 2014).

Coupled finite element electromagneticthermal modelling offers an elegant method for temperature evolution through different parts of the UGC starting from current conservation principle until thermal equilibrium of heat transfer. Hence, accurate estimation of thermal distribution at any point through the modelled domain. Therefore, the simulation results are subjected to extensive comparison with the IEC standard 60287 (1994) in order to ensure the validity of this modelling.

Figure 3 shows temperature evolution at two times inside the cable and the soil until thermal equilibrium is achieved at $\sim 3000 \mathrm{~h}$ of simulation for the reference case in this work. However, the approximately constant temperature of all conductors refers to constant transferred power throughout each current cycle.

Figure 4 illustrates the evolution of temperature profiles until steady-state temperature occurs at different layers in the cable for temperature dependent conductivity $(\sigma=\sigma(T))$ of the metallic parts compared with the IEC standard calculations for dynamic rating of UGC summarized in Anders (2005; and Eladawy (2017). The imposed rms current value of 431 A generates a steady-state temperature of $84.4^{\circ} \mathrm{C}$ at the conductor surface at $3000 \mathrm{~h}$ from current application for a $100 \%$ load factor, which is lower than the maximum continuous temperature, $\left(90^{\circ} \mathrm{C}\right)$ (Moore 1997). There is a decrease in conductor temperature by about $11 \%$ when considering constant conductivity $(\sigma=$ constant) of the metallic parts. However, the IEC standard 60287 (1994) calculation shows a conductor steady-state temperature of $80.46^{\circ} \mathrm{C}$ which is $4.6 \%$ lower than the calculated temperature with the finite element model as shown in Fig. 4.

Therefore, the steady-state temperature severely depends on the applied current, temperature dependent alternating current resistance of the metallic parts, number of phases, thermal resistance of each constructive domain and loss factors of metallic sheaths. The transient part of temperature profile highly depends on the soil thermal diffusivity, burial depth and heat capacitance of constructive metallic parts of the cable (Anders 2005). Consequently, this complete signature of the coupled electromagnetic-thermal behaviour provides designers and manufactures with the ability to study multiple operating and environmental conditions, and estimate UGC lifetime.

\subsection{Relative Permeability of Armour Layer}

Magnetic field distribution and losses in armour material have attracted the interest of many researchers, e.g. Anders (2005); and Lucca (2016) since the relative permeability of armour material may disturb the emanated magnetic field distribution. However, the relative permeability has different values from different cables' manufacturers depending on the type of the applied armour material (SAOG).

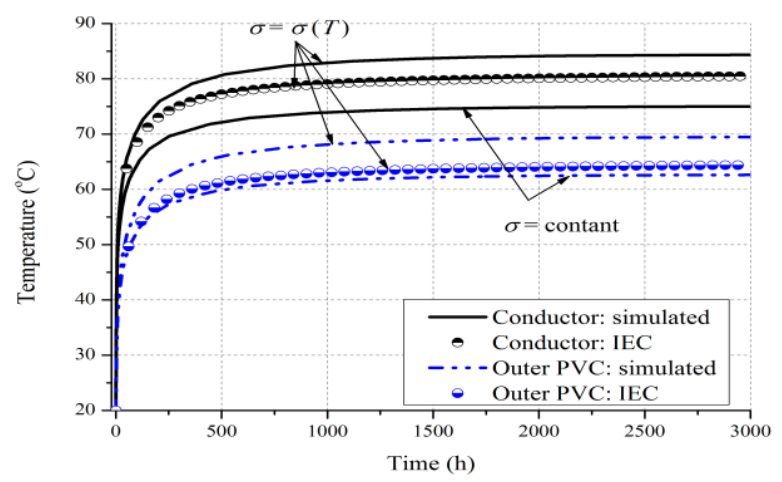

Figure 4. Temperature evolution profiles for different points in the core and outer points of PVC jacket until steady-state after $3000 \mathrm{~h}$ of simulation compared with the IEC standard calculation. 
Generally, most cable manufacturers take the relative permeability of 300 (Moore 1997; SAOG). The larger relative permeability of steel armour is, the lower magnetic field outside the cable (Lucca 2016). In addition, the larger the relative permeability of steel armour, the larger is the induced current density due to high intensification of magnetic field inside the highly permeable material and consequently reducing it in the neighbouring regions.

In reference case $\left(\mu_{r}=500\right)$, a maximum flux density of $0.22 \mathrm{~T}$ is observed in the steel armour near the closest individual copper sheaths due to this high-imposed relative permeability. The flux density on the conductor surface reaches a value of $0.02 \mathrm{~T}$ where its value has $2.07 \mu \mathrm{T}$ at the ground surface. It is worth mentioning that the latter value reaches up to $13.4 \mu \mathrm{T}$ when $\mu_{r}=1$ for the armor layer. Inside the touching domain of individual copper sheaths, the current density seems to be constant since the magnetic flux is the resultant of three balanced equidistance-varying fields. Calculation of losses in armour is not straightforward which depends on its material, cable type and installation method. The relative permeability $\left(\mu_{r}\right)$ of steel tape armour in Fig. 1 is changed without making any modifications of geometrical dimensions (Table 1) or physical parameters of constructive materials (Table 2).

Figure 5 shows the effect of relative permeability of steel armour on the temperature rise at different points inside the cable at steady state after $3000 \mathrm{~h}$ of simulation. For a nonmagnetic steel armour $\left(\mu_{r}=1\right)$, the steady-state temperature at the conductor surface reaches $75.8^{\circ} \mathrm{C}$ and continuously increases with the increase of relative permeability up to 500 by $11.35 \%$.

It is clear that the entrapment of magnetic field inside the cable leads to a significant rise of temperature at different layers of the cable because of increasing current density distribution and consequently power losses inside the metallic parts of the UGC.

\subsection{Single-Phasing Operation}

When the three-phase UGC is connected to a three-phase induction motor, unintended fault removal of one phase leads to a single phase running of the machine with drawing a greater current than the winding rated one. This type of fault may be due to motor's open-winding as result of burned connection, open circuit fault of fused contactors in the transformer-motor connection, fuse blowing, ...etc.

In oil industry (Metwally et al. 2008), it is common to keep running the electric submersible pumps (ESP) even if one phase of the motor is damaged or one of the fuses is blown out to keep oil production to the last minute before a complete failure of the motor. These failures have a very high cost because electric submersible pump's failures result in oil production losses or what is so-called "oil deferment" affecting revenue, additionally pulling out the motor from down-hole requires mobilization of a rig, which has a very high renting cost. However, phase removal represents the worst case of unbalanced current condition, which leads to a current increase by a factor of $\sqrt{3}$ in the remaining two phases of the UGC (Metwally 2010). It is found that current unbalance gives low contribution of power losses, and hence it is ignored in this paper. Irrespective of the overcurrent and thermal protection procedures, this single-phasing hazard may not be discovered until the burning of either the UGC or the running ESP occurs.

The traditional analytical method for UGC thermal rating (Anders 2005; Sedaghat 2014) may fail to predict the temperature level with the desired accuracy in the remaining phases of single phasing operation mode due to the complexity of thermal insulation resistance and parameter calculations. The coupled electromagnetic-thermal model offers a simple way of predicting this thermal performance due to single phasing of UGC by removing a current from one phase and changing the rated current of the other two phases.

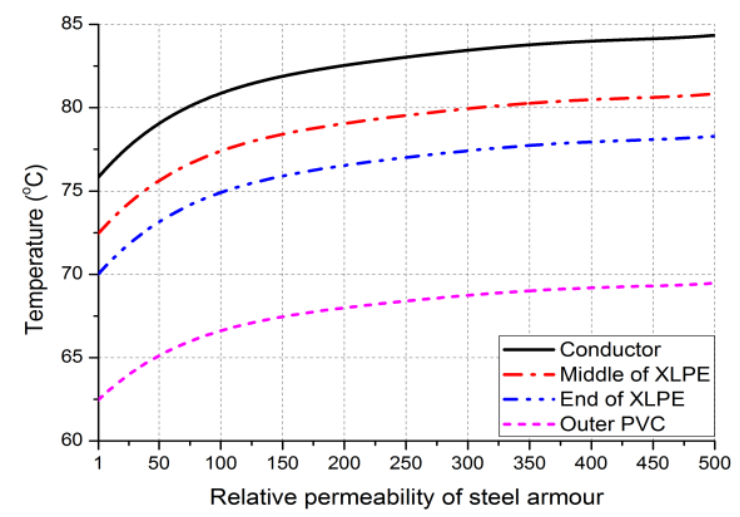

Figure 5. Effect of relative permeability of steel armour on the steady-state temperature rise inside different parts of the UGC. 
However, the simulation results show that when increasing the rated current by a factor of $\sqrt{3}$ as a result of phase removal, it would result in high conductor temperature values which exceed the maximum permissible value. This is due to the exponential increase in conductor temperature with increasing the conductor current. Hereby, the lower left conductor current of Fig. 1 is relaxed and the remaining two conductors are loaded by $0.5 \sqrt{3} I_{n}$ phase currents with $120^{\circ}$ of phase shift.

Figure $6 \mathrm{a}$ shows the resistive losses distribution inside the metallic parts of the UGC single-phasing operation. The maximum observed magnetic flux density in the steel armour is $0.26 \mathrm{~T}$ and it is located on steel periphery containing the two energized cores. The maximum induced current density and consequently resistive losses in the copper sheaths of energized conductors are increased to $\sim 247 \%$ and $\sim 662 \%$ of their values at a half rated current operation, respectively. From Fig. 6a, it can be seen that the energized conductors and their copper sheaths have these higher values of current density and resistive losses. Fig. 6b shows a comparison of steady-state temperature between normal operations of three-phase UGC at a half rated current and a single-phasing operation. All the three conductors attain a value of $34.5^{\circ} \mathrm{C}$ temperature at a steady state in case of normal operation with a half load current. In case of a single-phasing operation, the temperature of the de-energized conductor is $56^{\circ} \mathrm{C}$ because of the induced current density by the proximity effect, while it reaches up to $60.1^{\circ} \mathrm{C}$ at the surface of the upper core conductor, which is lower than the permissible temperature value for the other energized conductors. Any increase of the operating current in a single-phasing case would result in overheating of the insulation material and hence abrupts failure of the UGC (Metwally et al. 2008).

\subsection{Current Harmonics}

Current harmonic distortion can be generated by power electronic devices, which have been widely penetrated into industry. The preliminary effect of this disturbance is the introduction of additional power losses in UGC, and hence increases its operating cost and reduces its lifetime due to additional thermal stresses (Rasoulpoor et al. 2017). Harmonic filter seems to be a simple solution and its higher cost limits its application. Therefore, this harmonic current perturbation must be accounted for the proper ampacity derating and accurate calculation of thermal loading of cable constructive insulation material (Tofoli et al. 2006). In the above simulation, a pure sinusoidal current waveform is assumed flowing through the conductors. In case of harmonic perturbation current, however, the rms current $I_{r m s}$ of the nonsinusoidal waveform is calculated as follows (Tofoli et al. 2006):

$$
I_{r m s}=\sqrt{\sum_{h=1}^{\infty} I_{h}^{2}}=I_{l} \sqrt{1+T H D_{i}^{2}}
$$

where $I_{1}$ is fundamental current component, $I_{h}$ is the current of harmonic order $h$, and $T H D_{i}$ is the current total harmonic distortion. Increasing the harmonic order would result in conductor resistance increase, and consequently power loss. The effect of superimposed harmonics on the sinusoidal current can be accounted for

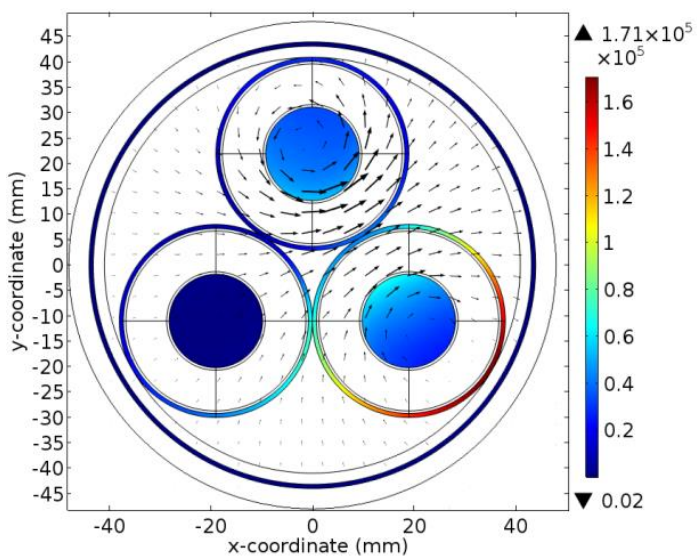

(a) Surface plot of resistive losses $\left(\mathrm{W} / \mathrm{m}^{3}\right)$ with proportional arrow direction of magnetic field intensity $(\mathrm{A} / \mathrm{m})$.

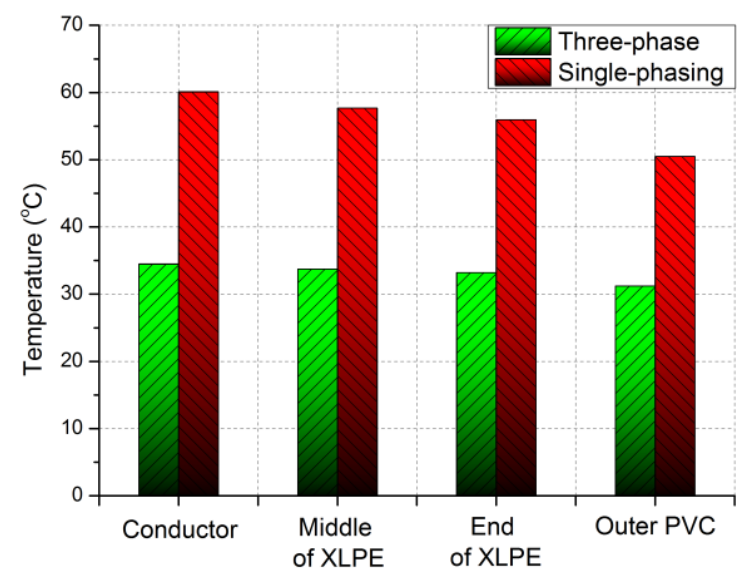

(b) Steady-state temperature at different layers inside the upper core and at the cable surface at half rated current for normal and single-phasing operations.

Figure 6. Single-phasing operation of UGC at half rated current. 
resistance calculation as follows (Tofoli et al. 2006):

$$
R_{a c}=R_{d c}\left(1+k_{s}+k_{p}\right)
$$

where, $R_{a c}$ and $R_{d c}$ are the ac and dc resistances at the operating temperature in $\Omega / \mathrm{km}$, and $k_{s}$ and $k_{p}$ are the skin and proximity effect, respectively. The skin effect parameter $x$ can be calculated as the following (IEC 60287 1994; Tofoli et al. 2006):

$$
x=0.01528 \sqrt{\frac{f \cdot \mu}{R_{d c}}}
$$

where, $f$ is the frequency in $\mathrm{Hz}, \mu$ is the magnetic permeability of the conductor. The skin and proximity effect parameters can be approximated as follows Tofoli et al. (2006):

$$
\begin{aligned}
& k_{s e}=10^{-3}\left(\begin{array}{l}
-1.04 x^{5}+8.24 x^{4} \\
-3.24 x^{3}+1.447 x^{2} \\
-0.2764 x+0.0166
\end{array}\right) \text {, if } x \leq 2 \\
& k_{s e}=10^{-3}\left(\begin{array}{l}
-0.2 x^{5}+6.616 x^{4} \\
-83.343 x^{3}+500 x^{2} \\
-1061.9 x+769.63
\end{array}\right) \text { if } 2<x \leq 100 \\
& k_{p e}=k_{s e}\left(\frac{d_{c}}{s}\right)^{2}\left(\frac{1.18}{k_{s e}+0.27}+0.312\left(\frac{d_{c}}{s}\right)^{2}\right)
\end{aligned}
$$

where, $d_{c}$ is the conductor diameter and $s$ is the axial spacing between conductors.

Metallic sheath and armour losses in XLPE UGC are about $5 \%$ of the three-conductor losses and can be ignored in per-unit power loss calculations (Gandhare et al. 2013). Considering only odd harmonics, the power loss $P$ in all conductors can be calculated as follows (Gandhare et al. 2013):

$$
P=3 I_{1}^{2}\left(R_{a c(1)}+\sum_{h=3}^{\infty} H_{h}^{2} R_{a c(h)}\right)
$$

where, $R_{a c(1)}$ and $R_{a c(h)}$ are the ac resistances for the fundamental and harmonic frequencies, respectively, and $H_{h}$ is the percentage of harmonic load.

Different cable sizes and conductor materials, namely copper and aluminium, are studied in order to investigate the effect of current harmonics on the power losses inside the UGC. The rating current for different cable sizes and conductor materials, namely copper and aluminium, are studied in order to investigate the effect of current harmonics on the power losses inside the UGC. The rating current for each copper conductor cable is applied on the same corresponding size of aluminium conductor cable. Figure 7 shows the variation of conductor resistance with the harmonic order for two different materials and for different cable cross-sectional areas. Increasing the harmonic order would result in increasing the conductor resistance, which depends on the cable dimensions due to both skin and proximity effects as observed by (9).

The harmonic spectrum of the three different types of pulse rectifiers, namely 6-, 12- and 18pulse rectifiers with current total harmonic distortion $T H D_{i}$ of $30.82 \%, 14.63 \%$ and $9.6 \%(\mathrm{Wu}$ et al. 2017), respectively, has been imposed through MATLAB program on the results of electromagnetic-thermal model to study the effect of current harmonics on the steady-state temperature rise of UGC. Figure 8 shows the effect of the studied harmonic distortion on the steady-state temperature rise for the considered different cable configurations.

It is worth mentioning that the greater the harmonic distortion, the larger is the number of harmonics. Consequently, each current harmonic generates its own individual heating effect. It can be seen that this increase in temperature depends on the harmonics signature of each type of rectifier and the rated current for each cable type. Increasing the 6pulse rectifier $T H D_{i}$ to $43.6 \%$ would result in

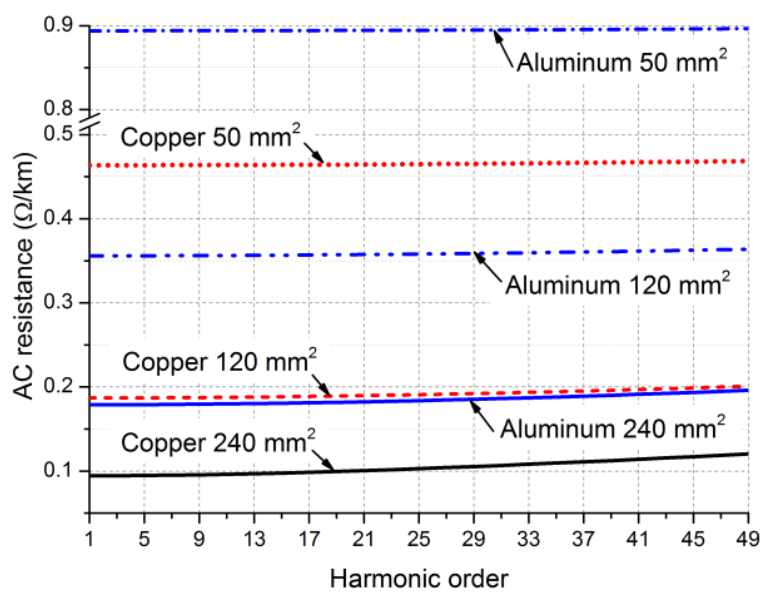

Figure 7. Effect of harmonic order on the ac resistance for different sizes and conductor materials of UGC. 
higher temperature as shown in Fig. 8. The 6pulse rectifier gives the highest conductor temperature rise for all considered cable configurations. Considering the pure sinusoidal waveform $\left(T H D_{i}=0\right)$ as the reference case of UGC $\left(240 \mathrm{~mm}^{2}\right)$, there is an increase of temperature by $\sim 10 \%$ and $\sim 19 \%, \sim 2 \%$ and $\sim 1 \%$ for 6- $\left(\mathrm{THD}_{i}=30.82 \%\right.$ and $\left.43.6 \%\right), 12$ - and 18 pulse rectifiers, respectively. In addition, the conductor temperature exceeds the maximum continuous conductor temperature in case of having 6-pulse rectifier. Therefore, the operating current must be decreased in order to meet the thermal limit of UGC, which results in cable derating due to this current harmonics distortion.

\subsection{Cable Lifetime}

UGC lifetime is one of utilities' major aspects in real operating conditions (Zhoun et al. 2017). It is mainly detected by the thermal stresses of the insulation material (Zarchi et al. 2016). Normally, cable lifetime $t_{\text {life }}$ is not affected when the conductor temperature is lower than the maximum continuous conductor temperature. However, it exponentially decreases when the temperature rises above its maximum limit according to Arrhenius Model as follows (Gandhare et al. 2013):

$t_{\text {life }}=t_{0} \exp \left(\frac{w_{a}}{k}\left(T^{-1}-T_{0}^{-1}\right)\right)$

where $T_{0}$ is the reference maximum continuous conductor temperature $(363.15 \mathrm{~K}), T$ is the accelerated ageing temperature in $\mathrm{K}, w_{a}$ is the activation energy for thermal degradation of XLPE $\left(w_{a}=1.1 \mathrm{ev)}\right.$ (Zarchi et al. 2016), $k$ is Boltzmann's constant in ev $/ \mathrm{K}$ and $t_{0}$ is the cable lifetime at $T=T_{0}$.

Figure 9 shows the expected per-unit lifetime of the UGC under different operating conditions when increasing the current rating and current total harmonic distortion. In the three-phase operation of the reference cable (240 $\mathrm{mm}^{2}$ ) and for pure sinewave, overloading exponentially decreases the lifetime. In the case of the single-phasing operation, the sharp rise of temperature rapidly degrades the cable above half of its current rating. In the case of the rated current for the 6-pulse rectifier $\left(\mathrm{THD}_{i}=30.82 \%\right)$, the cable lifetime is reduced to $\sim 43 \%$ and $\sim 49 \%$ of its reference lifetime for the 240 and $50 \mathrm{~mm}^{2}$ copper conductor cables, respectively. Therefore, the cable derating factor is necessary depending on the $T H D_{i}$ loading of the cable in order to meet the thermal limits of its constructive insulation material and consequently its lifetime. This issue will be discussed in the next Section.

\subsection{Cable Derating Factor due to Current Harmonics}

Cable derating factor $\left(k_{d}\right)$ can be calculated when the conductor temperature exceeds its maximum continuous value, which may be due to current harmonic distortion. In this study, for a known harmonic signature, $k_{d}$ is defined as the ratio of rms value of the current distorted by harmonic $\left(I_{r m s, d}\right)$ to the rms value of a pure sinusoidal current $\left(I_{r m s, p}\right)$ with fundamental frequency which produces the same losses as follows (Tofoli et al. 2006):

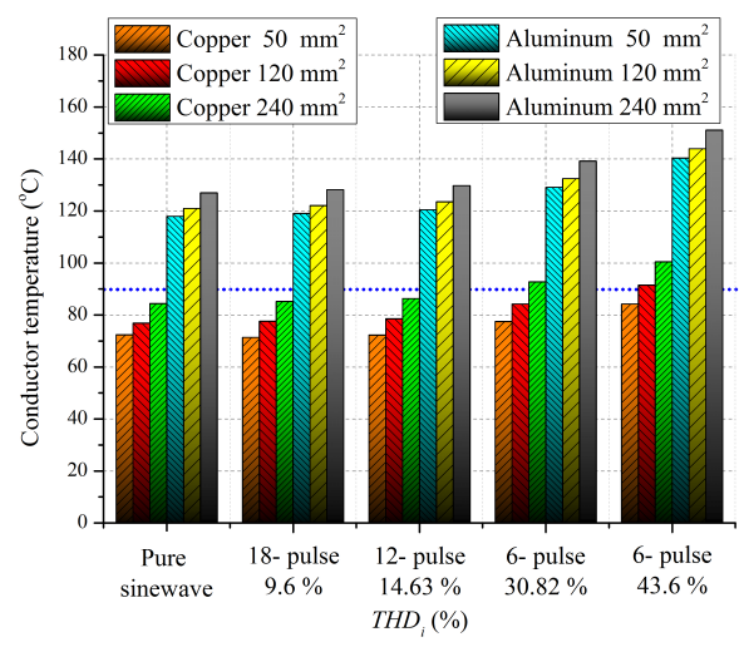

Figure 8. Effect of current total harmonic distortion of the steady-state conductor temperature rises for different sizes and UGC materials

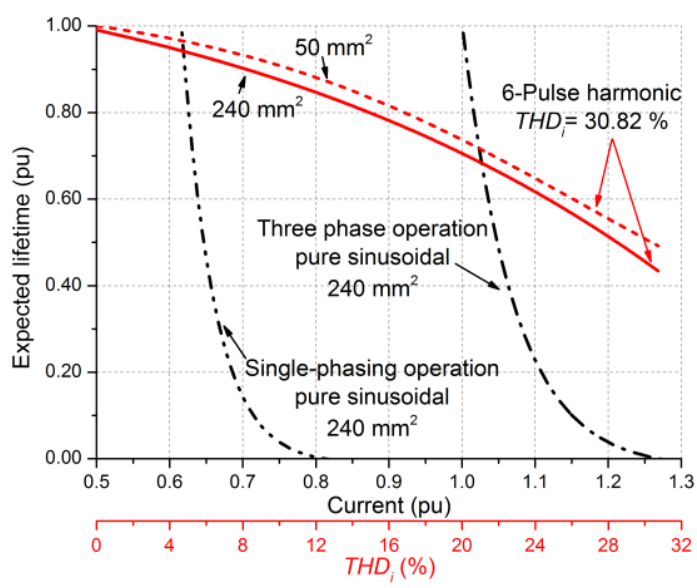

Figure 9. Expected per-unit lifetime of the cable under different operating conditions versus current rating and $\mathrm{THD}_{\mathrm{i}}$. 


$$
k_{d}=\frac{I_{r m s, d}}{I_{r m s, p}}=\sqrt{\frac{R_{a c(1)} / R_{d c}}{\sum_{h=1}^{\infty} H_{h}^{2} \frac{R_{a c(h)}}{R_{d c}}}}
$$

As long as the conductor temperature is lower than the maximum continuous temperature, there is no derating of cable's ampacity $\left(k_{d}=1\right)$. For the case of reference cable $\left(240 \mathrm{~mm}^{2}\right)$ and 6-pulse rectifier with $T H D_{i}=$ $43.6 \%$, the calculated derating factor $\left(k_{d}=\right.$ $0.9136)$ as a result of higher temperature than the maximum continuous one. Figure 10 shows the effect of different current harmonic distortion of 6-pulse rectifier on the conductor temperature of $240 \mathrm{~mm}^{2}$ UGC with the corresponding derating factor. It can be noticed that exceeding maximum continuous conductor temperature would result in a higher derating process.

\section{Conclusion}

A coupled electromagnetic-thermal method based on finite-element modelling is introduced to investigate the behaviour of UGC under different operating conditions using COMSOL Multiphysics software. Distributions of magnetic field, current density, resistive losses and temperature inside different layers of the UGC are calculated. Some realistic parameters are studied, such as temperature-varying conductivity and relative permeability of steel armour.

A single-phasing operating condition is considered to be one of the severest unbalanced cases. It significantly raises the temperature of the cable insulation material even at half of its rated current. Precautions should be considered in the case of this unintended operation mode owing to the rapid increase of magnetic field perturbation and excessive rise to temperature inside the cable, and hence shortening the cable lifetime.

Current harmonics distortion can also give rise to temperature because of high-frequency contents, which increases the resistance and consequently steady-state temperature. Higher steady-state conductor temperatures are expected in cables with larger conductor crosssectional areas, using aluminium core rather than copper, or using 6-pulse rectifiers rather than higher pulse types. Expected lifetime exponentially decreases with an increase above maximum continuous conductor temperature due to accelerated thermal aging. Therefore, it is tremendously important to limit the order and the percentage contents of the load current harmonics to reduce the harmonic loss and the harmonic temperature rise. Finally, cable derating process should be taken into consideration in order to meet the thermal limits of insulation material.

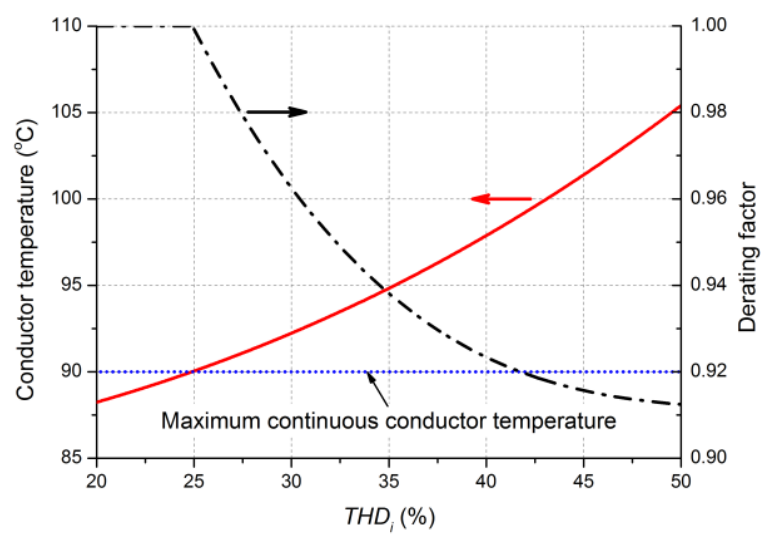

Figure 10. Effect of current total harmonic distortion on the conductor temperature and the corresponding derating factor for the $240 \mathrm{~mm}^{2}$ UGC.

\section{Conflict of Interest}

The authors declare no conflicts of interest.

\section{Funding}

No funding was received for this research.

\section{References}

Anders GJ, Napieralski A, Kulesza Z (1999), Calculation of the internal thermal resistance and ampacity of 3-core screened cables with fillers. IEEE Trans. Power Deliv, 14(3): 729734.

Anders GJ (2005), Rating of electric power cables in unfavorable thermal environment. (Wiley-IEEE press).

COMSOL Group Ltd., Stockholm, Sweden (20133), COMSOL multiphysics software package Version 4.4.

Eladawy M (2017), Dynamic derating of threephase underground power cable under current harmonic distortion. 19th International Middle East Power System Conference MEPCON'19. 
Gandhare WZ, Patil KD (2013), Effects of harmonics on power loss in XLPE cables. Energy and Power Engineering 5(4B): 12351239.

Gouda OE, Amer GM, El Dein AZ (2010), Effect of the formation of the dry zone around underground power cables on their ratings. IEEE Trans. Power Deliv, 26(2): 972-978.

Huang Z, Pilgrim JA, Lewin P, Swingler S, Gregory TG (2015), Thermal modelling and analysis for offshore submarine high-voltage direct current cable crossings. IET Gener. Transm. Distrib, 9(16): 2717-2723.

International Standard IEC 60287 (19944), Electric cables - calculation of the current rating - Part 1-1: current rating equations (100\% load factor) and calculation of lossessection 1: general. 1994-12.

Kovetz A (1990), The principles of electromagnetic theory. Cambridge University Press, 1990.

Lucca G (2016), Electromagnetic interference at power frequencies: shielding factor related to an urban environment. IET Sci. Meas. Technol. 10(6): 614-620.

Metwally IA, Gastli A (2008), Correlation between eddy currents and corrosion in electric submersible pump systems. Int. J. Thermal Sciences (IJTS), 47(6): 800-810.

Metwally IA (2010) Electrostatic and magnetic field analyses of $66-\mathrm{kV}$ cross-linked polyethylene submarine power cable equipped with optical fiber sensors. Electric Power Components and Systems 38(4): 465-476.
Metwally IA (2012), The evolution of medium voltage power cables. IEEE Potentials, 31(3: 20-25.

Moore GF (1997), Electric cables handbook. (Blackwell Science, 3rd edn.).

Oman Cables Industry (SAOG): Medium voltage cables. OCI/PBMVC/REV. 001/010410, Available: Www. omancables.com.

Rasoulpoor M, Mirzaie M, Mirimani SM (2017), Effects of non-sinusoidal current on current division, ampacity and magnetic field of parallel power cables. IET Sci. Meas. Technol, 11(5): 553-562.

Sedaghat A, de León F (2014), Thermal analysis of power cables in free air: Evaluation and improvement of the IEC standard ampacity calculations. IEEE Trans. Power Deliv, 29(5): 2306-2314.

Tofoli FL, Sanhueza SMR, de Oliveira A (2006), On the study of losses in cables and transformers in nonsinusoidal conditions. IEEE Trans. Power Deliv, 21(2): 971-978.

Wu B, Narimani M (2017), High-power converters and AC drives. (Wiley-IEEE Press, 2nd edn.).

Zarchi DA, Vahidi B (2016), Optimal placement of underground cables to maximise total ampacity considering cable lifetime. IET Gener. Transm. Distrib, 10(1): 263-269.

Zhoun C, Yi H, Dong X (2017), Review of recent research towards power cable life cycle management. IET High Voltage 2(3): 179 187. 